\title{
Servicio de internación domiciliaria para pacientes con enfermedades agudas
}

\author{
Juan Roubicek, ${ }^{1}$ Adrián Salvatore, ${ }^{1}$ Gabriela Kavka ${ }^{1}$ \\ y Carlos Wiersba ${ }^{1}$
}

RESUMEN El objetivo de este trabajo es presentar los resultados de 26 meses de actividad (enero de 1996 a febrero de 1998) del Servicio de Internación Domiciliaria Diferenciada de Agudos. Este servicio funcionó para 20 camas domiciliarias con dos equipos, cada uno formado por un médico y una enfermera, con una modalidad de atención similar a la de una sala de internación hospitalaria. Se evaluaron las características de la población internada, sus trastornos, las vías de administración de medicamentos, la satisfacción del grupo cuidador, los índices de rendimiento y los costos del sistema. Un total de 1789 pacientes tuvieron internación domiciliaria en ese período, con una estancia mediana de 4 días. El 76,5\% ingresaron desde la internación hospitalaria. Los trastornos cardiorrespiratorios fueron los más frecuentes $(45,5 \%)$ y el porcentaje de pacientes con enfermedad en fase terminal alcanzó 14,2\%. La vía de administración de medicamentos fue oral en $74 \%$ de los pacientes y parenteral en $26 \%$. La satisfacción de los pacientes fue muy buena y el costo de la internación se redujo a 70\% del costo de la internación hospitalaria. La participación familiar fue fundamental en esta modalidad de atención.

La internación hospitalaria suele ser física y emocionalmente traumática para el paciente y para la familia $(1,2)$ y también es costosa para el sistema de salud $(3,4)$. El Hospital Privado de la Comunidad de Mar del Plata (HPC) es un hospital general para la atención de enfermedades agudas, con más de 25 años de experiencia. Cuenta con 220 camas de hospitalización, atiende a una población de más de 100000 pacientes y tiene un total de egresos anuales de 14000 pacientes con una estancia promedio de 4,3 días (5). En este marco, en diciembre de 1995 se creó el

\footnotetext{
Hospital Privado de Comunidad de Mar del Plata, Servicio de Internación Domiciliaria, Argentina. Toda correspondencia debe dirigirse a Juan Pablo Roubicek. Dirección postal: Jujuy 4342 2b 7600Mar del Plata, Argentina. Teléfono/fax: 54-22399000 int. 233. Correo electrónico: ianroubi@ satlink.com
}

Servicio de Internación Domiciliaria Diferenciada de Agudos (SID). El objetivo de este trabajo es presentar los resultados de esta nueva modalidad para el cuidado de pacientes con enfermedades agudas durante el período de enero de 1996 a febrero de 1998.

\section{MATERIALES Y MÉTODOS}

El SID cubre un área geográfica de $104 \mathrm{~km}^{2}$ en la zona urbana de la ciudad de Mar del Plata y tiene una capacidad de 20 camas disponibles por día de internación extrahospitalaria. Cuenta con cuatro médicos de dedicación exclusiva, tres enfermeras profesionales $y$ una trabajadora administrativa.

Los criterios de admisión de los pacientes fueron que hubiera una indicación médica de internación pero sin la necesidad inminente de cuidados intensivos; que el entorno social fuera adecuado (con la presencia de familiares o cuidadores responsables), y que el paciente y cuidador aceptaran participar en el sistema de internación domiciliaria.

A diario se constituían dos equipos de atención, cada uno con un médico y una enfermera, que evaluaban a los pacientes en sus domicilios una o dos veces por día según lo exigiera la atención o el tratamiento. La revista diaria de los pacientes fue similar a la de una sala hospitalaria típica y abarcó el interrogatorio y examen físico de cada paciente, así como medidas e instrucciones para su tratamiento. En el caso de enfermos con tratamientos administrados por vía parenteral, estos se administraban durante la visita. La medicación era dispensada diariamente por la farmacia del hospital y el equipo médico la entregaba a cada 
paciente. Las muestras para los estudios complementarios de laboratorio se tomaron en el domicilio y se procesaron en el hospital, mientras que los estudios por imágenes se realizaron en el hospital de base. El transporte del equipo de salud estuvo a cargo del SID (unidad móvil y viáticos), mientras que los traslados eventuales del paciente estuvieron a cargo de ambulancias del Hospital Privado de la Comunidad (HPC).

El orden de visita de los pacientes se determinaba según la necesidad de administrar medicamentos por vía parenteral en horarios predeterminados; el surgimiento de consultas no programadas, solicitadas por vía telefónica, y la proximidad geográfica. Además, durante todo el día cada médico responsable del SID mantenía comunicación directa y eficaz con los pacientes por medio de teléfonos celulares de uso exclusivo.

Los datos de filiación de los pacientes, así como sus antecedentes médicos, diagnósticos de egreso, estudios complementarios, días de internación y fecha de egreso se anotaron prospectivamente en planillas especialmente diseñadas.

La continencia del grupo cuidador (definida como la capacidad y voluntad de la familia o cuidadores para atender a los requerimientos del paciente) fue evaluada subjetivamente por el médico tratante, con una escala ordinal de cuatro valores, desde mala a muy buena.

Se definió como intervención social a toda actividad que el equipo médico realizó en el domicilio del paciente, destinada a lograr la autosuficiencia del grupo cuidador a corto y largo plazo. Algunas de las intervenciones fueron la educación de los familiares de pacientes con enfermedades crónicas; la asignación de funciones a familiares o allegados para mejorar el bienestar del paciente, y el apoyo psicológico a familiares de pacientes en estadios terminales de enfermedades neoplásicas.

Con el propósito de conocer el grado de satisfacción de los egresados del SID, en mayo de 1996 se realizó una encuesta por vía telefónica, con una muestra de 78 pacientes que habían hecho uso del servicio en los 2 meses anteriores. Una trabajadora administrativa realizó el contacto telefónico con el propio paciente o sus familiares y registró el puntaje que estas personas le adjudicaron, en una escala analógica, al servicio en general, al personal médico y al de enfermería.

El análisis de costos corresponde a una estimación a partir de datos de los últimos 6 meses de 1997. Comprende solo los costos propios de la internación (honorarios del personal médico y de enfermería, medicación, material desechable, estudios complementarios y comunicaciones) y no los relacionados con la evolución posterior de los pacientes $u$ otros resultados a mediano o largo plazo.

El análisis estadístico de todos los datos se realizó con el programa Epi Info 6,04.

\section{RESULTADOS}

En el cuadro 1 se presentan algunas características de los 1789 egresados del SID, de enero de 1996 a febrero de 1998. Poco más de la mitad (52\%) eran mujeres; la edad media de los pacien- tes fue de 73,6 años (desviación estándar $\pm 12,9)$ y $84 \%$ tenían 65 años o más. Menos de $10 \%$ de los pacientes tuvieron afecciones crónicas de postración, tales como demencia avanzada o enfermedad crónica debilitante.

Cada equipo asistencial tuvo un promedio de siete pacientes diarios (un máximo de 10) y la duración promedio de cada visita fue de 42 minutos, con traslado incluido. Un promedio mensual de 69 pacientes (entre 54 y 114) egresaron del SID. La mediana de los días de internación fue de 4 (recorrido intercuartílico de 3 a 4) y la moda, de 2. Sin embargo, cabe mencionar que 20 pacientes superaron los 30 días de internación. El porcentaje de ocupación promedio de las 20 camas disponibles fue de $63 \%$.

Con respecto al seguro médico, 93\% de los pacientes fueron atendidos en el HPC por un sistema de capitación a través del Instituto Nacional de Servicio Social para Jubilados y Pensionados. Ingresaron al SID 76,5\% previa internación hospitalaria $(68,5 \%$ en el área clínica y $8 \%$ en la quirúrgica). Los demás pacientes tuvieron internación directa en el SID: $21 \%$ desde los servicios de emergencia, guardia domiciliaria y

\section{CUADRO 1. Características generales de 1789 pacientes egresa- dos del Servicio de Internación Domiciliaria. Hospital Privado de Comunidad de Mar del Plata, Argentina, enero de 1996 a febrero de 1998}

\begin{tabular}{lc}
\hline \multicolumn{1}{c}{ Variable } & $\%$ \\
\hline Seguro médico & \\
Instituto Nacional de Servicio Social para & 93,0 \\
Jubilados y Pensionados & 5,4 \\
Planes prepagados de salud del hospital & 1,6 \\
Obras sociales, otros prepagados y particulares & \\
Diagnósticos de egreso más frecuentes & 26,4 \\
Enfermedad respiratoria & 19,1 \\
Enfermedad cardio o cerebrovascular & 14,2 \\
Enfermedad neoplásica & 7,3 \\
Enfermedad cutánea (celulitis y abscesos) & 7,0 \\
Enfermedad endócrina o metabólica & \\
Modalidad terapéutica & 74,0 \\
Vía oral & 15,0 \\
Vía subcutánea & 11,0 \\
Vía endovenosa & Sobre 10 puntos \\
Encuesta de satisfacción (analógica) & 9,41 \\
Servicio en general & 9,73 \\
Personal de enfermería & 9,91 \\
Profesionales médicos & \\
\hline
\end{tabular}


atención domiciliaria de pacientes crónicos, y los restantes (2,5\%) desde consultorios externos.

En el mismo cuadro 1 se muestran los diagnósticos primarios de egreso. Entre ellos destacan los trastornos respiratorios, que representaron $26,4 \%$, incluidas la enfermedad pulmonar obstructiva crónica en fase aguda y la neumonía. Entre los trastornos cardiovasculares y cerebrovasculares predominó la insuficiencia cardíaca y entre los trastornos metabólicos, los hidroelectrolíticos y la diabetes descompensada. Cabe mencionar que los pacientes diabéticos se internaron, principalmente, para ajustar la dosis de la medicación hipoglucemiante a la realidad de sus hogares.

A $85 \%$ de los pacientes atendidos durante el período se les dio de alta con control ambulatorio o atención domiciliaria para pacientes crónicos. Hubo una mortalidad de $8 \%$ y el $7 \%$ restante fue remitido al hospital para su seguimiento intrahospitalario.

A $20,7 \%$ de los pacientes se les efectuaron uno o más análisis de laboratorio, en su mayoría exámenes de baja complejidad; a 3,6\% se les hicieron estudios radiológicos simples, y a menos de $5 \%$, una evaluación especializada por consulta.

Con respecto a las vías de administración de medicamentos (cuadro 1), se observó un uso creciente de vías parenterales: la subcutánea para cuidados paliativos e hidratación y la endovenosa para la administración prolongada de antibióticos (hasta tres dosis diarias) y diuréticos.

La continencia familiar se consideró muy buena en $56 \%$ de los casos; buena en $37,4 \%$, y regular en los casos restantes.

La encuesta de satisfacción mostró un puntaje promedio superior a 9 en una escala de 0 a 10 (cuadro 1) y todos los encuestados consideraron satisfechas sus expectativas.

Todos los pacientes tuvieron alguna intervención social realizada por el equipo tratante. La más frecuente fue la educación en la administración de los medicamentos y en los cuidados de pacientes con deterioro cognoscitivo, postración y diabetes.
Menos de 2\% requirieron la intervención de asistentes sociales a través de interconsultas.

El costo total de una cama del SID durante la ocupación promedio hallada en este estudio representó $70 \%$ del costo de una cama de internación hospitalaria en el servicio de clínica médica (SCM). A su vez, 70\% del costo promedio de una cama correspondió a gastos en personal, mientras que la medicación y el material desechable representaron 20\% (principalmente por los antibióticos parenterales y el clorhidrato de morfina).

\section{DISCUSIÓN}

El resurgimiento del cuidado de pacientes en el domicilio, en este caso de pacientes con enfermedades agudas, no es un fenómeno casual. Por el contrario, hay que ubicarlo en el contexto de una tendencia cada vez mayor en la medicina a cuidar la calidad de la vida, revalorizar al médico de cabecera, ofrecer cuidados paliativos y adoptar políticas acordes a las condiciones de salud de la población (6).

En este trabajo presentamos los resultados de dos años de experiencia con la nueva modalidad de internación domiciliaria. Sin embargo, a todos nuestros pacientes se les indujo a aceptar el SID como alternativa a la hospitalización, por lo que la muestra de pacientes no representa a quienes no aceptaron el sistema a pesar de estar en condiciones clínicas de ingresar. En este sentido, la selección de los participantes constituye el principal factor limitante de nuestro trabajo y no permite hacer comparaciones con el medio hospitalario. Por el contrario, este sesgo de selección podría explicar parcialmente que la continencia familiar haya resultado buena o muy buena en más de $90 \%$ de los casos.

Un modelo hipotético de internación domiciliaria publicado recientemente (7) propone una selección retrospectiva de pacientes con criterios similares a los que nosotros utilizamos. Sin embargo, los autores plantean un menor uso de recursos y una selección de pacientes con menos complicaciones.
El SID tuvo un promedio de 69 egresados al mes, lo que representó 5,6\% de los egresos mensuales del HPC. La media de los días de internación del HPC fue de 4,3 en su conjunto, al igual que en el servicio de clínica médica, y la mediana de los días de internación en el SID fue de 4 (consideramos que la mediana es el valor más representativo porque no se trata de una distribución normal). El hecho de que 20 pacientes hayan superado los 30 días de internación podría deberse a que eran enfermos con tratamientos endovenosos prolongados o con enfermedades terminales que fueron remitidos a nuestro servicio, lo cual implica, nuevamente, un sesgo de selección.

Los pacientes mostraron distinta afiliación al seguro médico que los egresados del resto del hospital. El porcentaje de pacientes provenientes de obras sociales fue mucho más bajo (16,8\% en el HPC; $8,9 \%$ en el SCM; y 1,6\% en el SID). Es probable que esta baja utilización se deba a la falta de convenios preestablecidos para esta prestación, que se ofreció individualmente a cada paciente, y a algunos retrasos en la aceptación y autorización por parte de algunos prestadores.

La mayoría de los pacientes ingresaron desde el SCM, lo cual no es de extrañar, dado que el servicio se creó como una extensión de la sala de internación del SCM y con médicos formados en esta área. Los ingresos desde los servicios quirúrgicos tuvieron su origen principalmente en la necesidad de administrar tratamientos prolongados con antibióticos a pacientes con complicaciones posquirúrgicas o con enfermedades terminales.

La edad media de los pacientes atendidos por el SID fue similar a la de los pacientes hospitalizados en el SCM (73,6 años y 74,3, respectivamente). También fueron similares sus enfermedades más frecuentes: las del aparato respiratorio ocurrieron, en el mismo orden, en $26,4 \%$ y $27,6 \%$ de los casos; las enfermedades neoplásicas, en $14,2 \%$ y $12,8 \%$. Como el SCM no interna pacientes con enfermedades cerebrovasculares - estos ingresan en el servicio de neurología-, el porcentaje de pacientes bajo los códigos de enfer- 
medades cardiovasculares y cerebrovasculares fue de $19,1 \%$ en el SID y de $14 \%$ en el SCM. La mortalidad de personas internadas en el SID (8\%) fue más baja que la de personas internadas en el SCM (13,6\%), pero similar a la de los pacientes del hospital en general $(7,9 \%)$.

El escaso uso de medios complementarios se debió a que la mayoría de los pacientes provenían de la internación hospitalaria y ya habían sido sometidos a los estudios pertinentes. Cuando los pacientes ingresaron desde el servicio de urgencias por lo general tenían únicamente los estudios mínimos indispensables.

En otros trabajos publicados la administración de medicamentos también se efectuó por vía subcutánea (8) y endovenosa (9). Sin embargo, en el SID no se requirieron dispositivos de alta tecnología para administrar los medicamentos, como se observa en los informes encontrados en la literatura $(10,11)$.

Fue difícil definir índices de rendimiento para una modalidad de atención que tiene solo algunos puntos de comparación con el medio hospitalario $\mathrm{y}$ pocas referencias en la literatura y donde predominan informes sobre la atención domiciliaria de pacientes crónicos. Por lo tanto, creemos que nuestros datos pueden ser un buen punto de partida para hacer comparaciones con otras experiencias similares.

De manera general, las tendencias fueron similares a las observadas en el resto del hospital, especialmente en el SCM. Los días de internación fueron similares en el hospital y en el SID, pero la mayoría de los pacientes de este último habían tenido una estancia hospitalaria previa, lo cual impide hacer una comparación rigurosa. El porcentaje de ocupación no fue óptimo durante el período estudiado (63\% en el SID frente a $77 \%$ en el HPC). Los índices de ocupación afectan a los costos del servicio, ya que estos dependen principalmente de la estructura montada para 20 camas; si hubiera mayor ocupación, el costo de la internación domiciliaria sería aun menor, ya que la principal inversión es en el pago al personal. Estudios aleatorizados, realizados con sistemas de "hospital en domicilio" con complejidad distinta de la del sistema que aquí se describe (más actividades de enfermería y rehabilitación y menos visitas médicas) mostraron ser sistemas eficaces en relación con los costos, con re- sultados análogos a los del hospital en general (12-14).

La satisfacción de nuestros pacientes parte de la confianza que se alcanzó en la relación entre médico y paciente, que se vio facilitada por el tratamiento de los pacientes en su propio entorno y sin la complejidad de un hospital. Además, probablemente las intervenciones sociales no hubieran podido proporcionarse ampliamente en el ámbito hospitalario, ya que en ellas participó un grupo familiar mucho más amplio del que habitualmente entra en contacto con los profesionales de salud en el hospital. Como se ha documentado, las visitas domiciliarias permiten identificar hasta dos nuevos problemas por paciente y pueden generar hasta ocho nuevas recomendaciones para su atención (4).

En conclusión, el Servicio de Internación Domiciliaria Diferenciada de Agudos se mostró apropiado para el cuidado de pacientes con enfermedades agudas. Su efectividad fue comparable a la del hospital general, si se tiene en cuenta el sesgo en la selección de pacientes. Fue un sistema de complejidad relativamente alta y bajo costo que enfocó al paciente en su totalidad biosocial.

\section{REFERENCIAS}

1. Creditor MC. Hazards of hospitalization of the elderly. Ann Intern Med 1993;118:219-223.

2. Coccaro EF, Prudic J, Rothpearl A, Nurenberg HG. Effect of hospital admission of DST results. Am J Psychiatry 1984;141:982-985.

3. Worobec G, Brown NK. Hypodermoclysis therapy in a chronic care hospital setting. J Gerontol Nurs. 1997;23(6):23-28.

4. Home Care in the 1990s. Council on Scientific Affairs [Review]. JAMA 1990:263;1241-1244.

5. Paz RA. La Fundación Médica Mar del Plata y el Hospital Privado de Comunidad. Medicina 1998;58:221-227.

6. Van Weel C. Primary care: favourite or scientific discipline? Lancet 1996;348:1431-1432.

7. Leff B A, Burton LA, Bynum JW, Harper MA, Greenough WB, Steinwachs D. Prospective evaluation of clinical criteria to select older persons with acute illness for care in a hypo- thetical home hospital. J Am Geriatr Soc 1997; 45:1066-1073.

8. Bruera E, Legris MA, Kuehn N, Miller MJ. Hypodermoclisis for the administration of fluids and narcotics analgesics in patients with advanced cancer pain. Cancer 1990;62: 15,407-411.

9. Gilbert DN, Dworkin RJ, Raber SR, Leggett JE. Outpatient parenteral antimicrobial-drug therapy. N Engl J Med 1997;337:13, 829-838.

10. Sheldon P, Bender M. High-technology in home care. An overview of intravenous therapy. Public Health Nurs 1990;7:130-137.

11. Bruera E. Ambulatory infusion devices in the continuing care of patients with advanced disease. J Pain Symptom Manage 1990;5:287-96.

12. Shepperd S, Harwood D, Jenkinson C, Gray A, Vessey M, Morgan P. Randomised controlled trial comparing hospital at home care with inpatient hospital care. I: Three-months of follow-up of health outcomes. BMJ 1998; 316:1786-1791.

13. Shepperd S, Harwood D, Jenkinson C, Gray A, Vessey M, Morgan P. Randomised controlled trial comparing hospital at home care with inpatient hospital care. II: Cost minimisation analysis. BMJ 1998;316:1791-1796.

14. Hugest SL, Cummings J, Weaver F, Manheim $\mathrm{L}$, Braun B, Conrad K. A randomised trial of the cost effectiveness of VA hospital-based home care for the terminally ill. Health Serv Res 1992;26:801-817.

Manuscrito recibido el 3 de septiembre de 1998 y aceptado para publicación, tras revisión, el 19 de mayo de 1999. 
ABSTRACT This study presents the results of 26 months of work, from January 1996 through February 1998, of the Distinct Home Hospitalization Service for Acute Patients. This service managed 20 home beds with two teams, each with a physician and a nurse, with

Home hospitalization for patients with acute illnesses a care approach similar to that for a room in a hospital. Among the items evaluated were the attributes of the admitted population, their illnesses, the form of administering drugs, the satisfaction of the caregivers, the indices of performance, and the costs with this approach. A total of 1789 patients had home hospitalizations over the period, with a median stay of 4 days. Of the patients, $76.5 \%$ were admitted from inhospital care. The most frequent illnesses were cardiorespiratory ones $(45.5 \%)$, and the proportion of patients with a terminal illness was $14.2 \%$. Drugs were administered orally with $74 \%$ of the patients and parenterally in $26 \%$. The patients' satisfaction level was very high, and the cost of the hospitalization was $70 \%$ of that for in-hospital care. Family involvement was key in this approach to care.

\section{ERRATUM}

Cecere, et al., " Trypanosoma cruzi infection in Triatoma infestans and other triatomines: Long-term effects of a control program in rural northwestern Argentina" (Rev Panam Salud Publica/Pan Am J Public Health 1999;5(6):392-399)

Se llama la atención de los lectores a un error de traducción que aparece en el resumen en castellano del artículo citado (p. 399). La quinta oración, que dice "T. guasayana y T. sordida fueron, respectivamente, ninfas y adultos recogidos en el área peridoméstica", debe decir "Los T. guasayanay T. sordida infectados fueron, respectivamente, ninfas y adultos recogidos en el área peridoméstica". Lamentamos el error, que ocurrió por entera responsabilidad de nuestra redacción.

Cabe añadir por solicitud de los autores que el término triatómido utilizado en el re sumen en español es de derivación dudosa y que el término correcto es triatomino, que proviene del latín Triatominae, subfamilia a la que pertenecen los redúvidos en cuestión. 